\title{
Optoelectronic switches based on diffusive conduction
}

\author{
Hilmi Volkan Demir ${ }^{a)}$ and Fatih Hakan Koklu \\ Department of Physics, Bilkent University, Bilkent, Ankara 06800, Turkey; Department of Electrical \\ Engineering, Bilkent University, Bilkent, Ankara 06800, Turkey; and Nanotechnology Research Center, \\ Bilkent University, Bilkent, Ankara 06800, Turkey
}

Micah B. Yairi, James S. Harris, Jr., and David A. B. Miller

Edward L. Ginzton Laboratory and Solid State and Photonics Laboratory, Stanford University, Stanford, California 94305

(Received 30 November 2005; accepted 24 June 2006; published online 18 August 2006)

\begin{abstract}
We study the process of diffusive conduction that we use in our optoelectronic switches to achieve rapid optical switching (on a picosecond time scale). We present the characteristic Green's function of the diffusive conduction derived for arbitrary initial conditions. We also report the series solutions of the diffusive conduction obtained for different boundary conditions $(V=0$ and $\nabla V=0$ along the device contact lines) in different device geometries (rectangular and circular mesas). Using these analytical results, we investigate the effect of boundary conditions on the switching operation and the steady state behavior in optical links. We demonstrate the feasibility of using such diffusive conductive optoelectronic switches to establish optical links in return-to-zero and non-return-to-zero coding schemes. For multichannel optical switching, we discuss possible use of a single optoelectronic switch to accommodate multiple channels at once, with $>100$ optical channels (with a $2000 \mathrm{~mm}^{-2}$ channel density and $<10 \%$ cross-talk), predicted on a $300 \times 300 \mu \mathrm{m}^{2}$ mesa with a device switching bandwidth of $>50 \mathrm{GHz}$, leading to a $5 \mathrm{~Tb} / \mathrm{s}$ aggregate transmission in principle. This approach of using multiple parallel channels on a single switch is completely opposite to the traditional idea of arraying many switches. This proposed scheme eliminates the need for on-chip switch integration and the need for the alignment of the optical channels to the integrated individual switches. () 2006 American Institute of Physics. [DOI: 10.1063/1.2234818]
\end{abstract}

\section{INTRODUCTION}

Today's high-speed optoelectronic systems including receivers and transmitters commonly employ high-speed optoelectronic diodes such as photodiodes and modulator diodes. These devices are typically designed and implemented as lumped circuit elements, where the entire device capacitance $(C)$ and its in-series resistance $(R)$ predominantly determine the operation speed. ${ }^{1-6}$ Such lumped optoelectronic devices are, thus, $R C$ limited. Typically, these lumped devices are constrained by their epitaxial growth and fabrication limitations. To circumvent this fundamental $R C$ time constant limit, an optoelectronic diode can alternatively be operated as a distributed $R C$ element, where its operation speed is set by its local device parameters such as capacitance per unit area and sheet resistance, rather than its lumped device parameters such as total device capacitance and parasitic contact resistance. Distributed behavior is achieved in a wellknown class of devices, traveling-wave devices (travelingwave electroabsorption modulators, traveling-wave waveguide detectors, etc.), though in those cases, it is more of an inductive-capacitive (LC) form, with propagating waves. $^{7-21}$

In this paper, we present another class of devices that rely on distributed $R C$ behavior via diffusive conduction for high-speed optical switching: diffusive conductive switches. $^{22-25}$ These devices completely eliminate the

${ }^{a)}$ Electronic mail: volkan@ bilkent.edu.tr lumped $R C$ limitation and thus operate much faster than their lumped counterparts. ${ }^{26-32}$ In the diffusive conduction based switches, as in traveling-wave devices, only the internal local device parameters set the device operation speed (for example, at $50 \mathrm{GHz}$ ). But, for the diffusive conductive switches, unlike traveling-wave devices, the high-speed operation does not require impedance matching (for example, at $50 \Omega$ ) between the device and the external circuitry. Furthermore, these diffusive conductive devices have the unique ability to operate locally within a small fraction of the device (using a device area of about a few micrometers in diameter) and thus accommodate multiple optical channels (possibly as large as 100 channels in principle) all on a single device, if desired.

In diffusive conductive switches, we carefully engineer the process of voltage diffusion so that, during the switching operation, the optically induced voltage profile, which is initially nonuniform across the lateral device plane, diffuses away into a flat voltage profile very rapidly (on a picosecond time scale). This process is a result of the electromagnetic wave propagation of the local voltage change across the twodimensional $R C$ device plane, driven by the spatial variations in the voltage profile. Our group has previously presented the diffusive conduction solution of a transient voltage profile for an instantaneous, spatially Gaussian, surface-normal light pulse incident on an infinitely large device plane. ${ }^{22}$ In this paper, we study the modeling and application of diffusive conduction for a variety of real-world initial and boundary device conditions in different device geometries. Also, we 


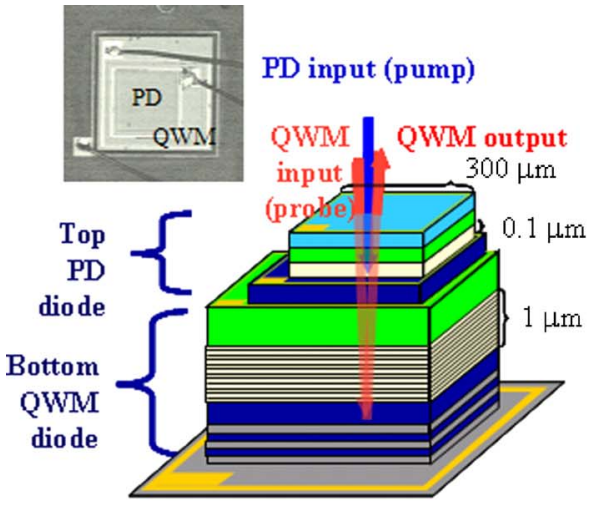

(a)

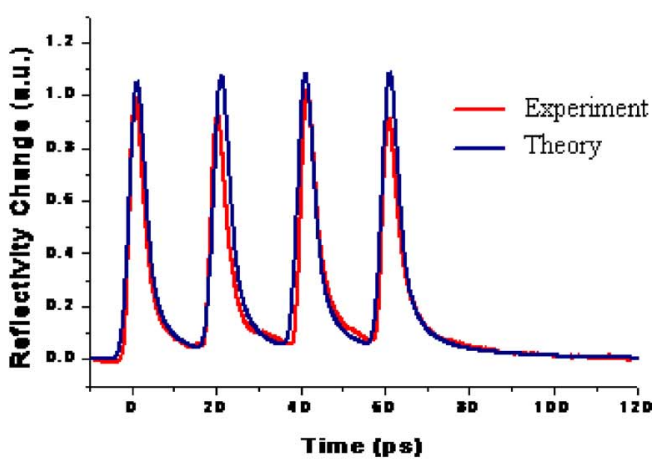

(b) compare the resulting device performances for these different cases and provide a perspective for their different system uses. Here, we base our switch modeling on a Green's function solution, characteristic of the diffusion process, and independent of the initial device condition. In rectangular and circular device geometries, we obtain series solutions for the special cases of Dirichlet and Neumann boundary conditions, for which the potential and its derivative along the device boundary are set to zero, respectively, corresponding to short-circuited and open-circuited device contact lines. Furthermore, we investigate the effects of diffusive conduction boundary conditions explicitly on the optical switching operation. Using these solutions, we examine a pulse-probe all optical switching process for a pulse train to show that an optical pulse stream always leads to a steady state device operating point that depends on the device speed and pulse repetition rate for the case of zero-potential boundary conditions.

Diffusive conduction allows for accommodation of $\mathrm{mul}$ tiple optical channels on a single device, facilitating the efficient use of the lateral device area with many channels in parallel. Taking advantage of this unique property, it may be possible to increase the aggregate transmission of a diffusive conduction based switch by many orders of magnitude to the $\mathrm{Tb} / \mathrm{s}$ range. Naturally, the optical channels affect each other, resulting in crosstalk between them. Calculated for different device parameters and channel densities (or, equivalently, channel spacing), we find the crosstalk between adjacent channels to be very low, possibly enabling the establishment of dense optical links. For example, a channel spacing of $<30 \mu \mathrm{m}$ yields $<10 \%$ crosstalk, which means that $>100$ channels could be accommodated on a typical 300 $\times 300 \mu \mathrm{m}^{2}$ device with a switching bandwidth of $50 \mathrm{GHz}$, leading to an aggregate transmission of $5 \mathrm{~Tb} / \mathrm{s}$. Our approach of using multiple channels on a single device is completely opposite to the traditional idea of arraying many switches on a chip. As a result, this scheme conveniently eliminates the need for switch integration and the need for the alignment of the optical channels to the individual switches integrated on the chip.

In this paper, we will discuss the process of diffusive conduction in greater depth in the context of optical switching. First, in Sec. II, we will briefly review the optoelectronic switch and its operation. Subsequently, in Sec. III, we will present solutions to the diffusive conduction derived for realcase operating device conditions and geometries and present a sample of simulation results to show the effect of device conditions on the voltage diffusion process and optical switching operation. Finally, in Sec. IV, we will discuss the use of diffusive conduction based optoelectronic switches to set up single and multiple optical links and demonstrate the feasibility of establishing optical links in return-to-zero and non-return-to-zero data coding schemes and dense parallel optical links on a single diffusive conduction based switch.

\section{DIFFUSIVE CONDUCTION BASED SWITCH OPERATION}

We have demonstrated different generations of optoelectronic switches based on diffusive conduction. ${ }^{22,24,25}$ Among these, in this paper, we focus on the surface-normal architecture of a vertical dual-diode switch structure shown in Fig. 1(a), with the top diode serving as the photodetector and the bottom diode as the modulator. Both of the diodes integrated in the switch structure use a $p-i$-n configuration, with the intrinsic region of the bottom modulator diode comprising multiple quantum wells (MQWs). We use two optical inputs to the switch, one as the control (pump) signal and the other as the probe signal. The pump signal is an input to the top photodiode, whereas the probe signal is an input to the bottom modulator diode, also sketched in Fig. 1(a). The top diode is strongly reverse biased, and the bottom diode is slightly reverse biased such that the MQWs of the bottom diode are transparent to the probe pulse when there is no control pulse. The top diode, while always transparent to the probe pulse, is always opaque to the control pulse. When a control beam is incident on the device, it is absorbed in the intrinsic region of the top diode. Its photogenerated carriers then separate across the instrinsic region of the top diode due to its reverse bias and give rise to voltage screening in the vicinity of the control beam. This results in a similar local voltage change in the bottom diode due to the capacitive coupling between the diodes. Consequently, the optical absorption of the MQW region in the bottom diode temporarily changes, becoming absorbing for the probe pulse through the quantum confined Stark effect (QCSE). ${ }^{33}$ Finally, the optically induced voltage decays away through diffusive conduction and the device recovers for the next pulse. Conse- 
quently, using the voltage dynamics of the diode, it is possible to transfer input data (pump) onto another beam (probe), when the probe is incident on the same area. Figure 1(b) shows the experimental switching operation for a $50 \mathrm{GHz}$ burst logic pulse train with 20 ps repetition period, plotted along with our theoretical simulation of the device based on the diffusive conduction solution. There is a strong agreement between the experimental data and our diffusive conduction based model. A more detailed description of the switch structure and its characterization can be found in the work of Yairi et al. ${ }^{25}$

\section{DIFFUSIVE CONDUCTION PROCESS}

Diffusive conduction, also referred to as enhanced diffusion, was described by Livescu et al. ${ }^{31}$ This mechanism was utilized for optically controlled modulation and switching. ${ }^{22}$ It was also shown that the diffusive conduction mechanism is the same as the mechanism known as giant ambipolar diffusion. $^{26}$

Diffusive conduction is an extension of the voltage dynamics of a dissipative transmission line, where a voltage pulse travels much faster than individual electrons in ordinary inductive-capacitive transmission lines. The switch structure we study here is a dissipative transmission plane where the series resistances of the $p$ and $n$ layers dominate over the inductive impedance, resulting in dissipative transmission. Yet still the dissipative wave propagation is much faster than individual electron motion.

The mathematical description of the voltage diffusion is given by the partial differential equation of

$$
\nabla_{x y}^{2} V(x, y, t)=\frac{1}{D} \frac{\partial V(x, y, t)}{\partial t} .
$$

Here, $D$ is the diffusion constant defined as $D=\left(R_{\mathrm{sq}} C_{A}\right)^{-1}$, where $R_{\mathrm{sq}}$ is the effective resistance per square of the conducting $p$ and $n$ layers, and $C_{A}$ is the capacitance per unit area. Equation (1) was previously solved for an infinite $x y$ plane with a Gaussian voltage profile imposed as an initial condition in particular. ${ }^{22}$ Here we derive a general Green's function solution for the case of arbitrary initial conditions with no boundary conditions:

$$
V(x, y, t)=\int_{-\infty}^{\infty} \int_{-\infty}^{\infty} d x^{\prime} d y^{\prime} f\left(x^{\prime}, y^{\prime}\right) G\left(x-x^{\prime}, y-y^{\prime}, t\right),
$$

where $f(x, y)=V(x, y, t=0)$ and

$$
G(x, y, t)=\frac{1}{4 \pi D t} \exp \left(-\frac{x^{2}+y^{2}}{4 D t}\right) .
$$

Note that in Eq. (2), we express the general solution $V(x, y, t)$ as a convolution of a characteristic Green's function $G(x, y, t)$ with an arbitrary initial condition $f(x, y)$.

In addition, here we also present the solution results of the diffusion equation derived for two different device boundary conditions: $V=0$ (as a special case of Dirichlet boundary condition) and $\nabla V=0$ (as a special case of Neumann boundary condition) along the device boundary at all times. These boundaries physically correspond to the device contact metal lines that connect to the external driving cir-
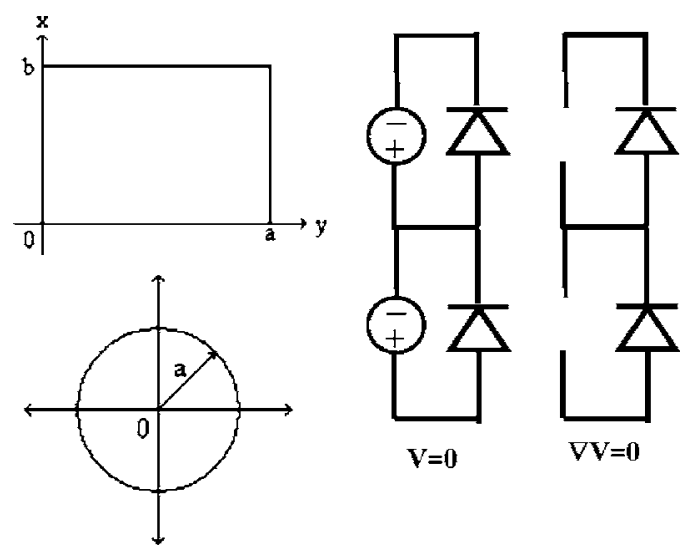

FIG. 2. Generic rectangular and circular device geometries defined to impose $V=0$ boundary conditions along their device perimeters when the integrated switch is dc biased across the detector and the modulator, and to impose $\nabla V=0$ boundary conditions when not biased.

cuitry. Therefore, $V=0$ boundary condition is the mathematical equivalent of a short-circuited device contact line, whereas $\nabla V=0$ boundary condition is that of an open-circuit one.

When solving the diffusion equation with respect to $V$ $=0$ boundary conditions on rectangular device geometry, as sketched in Fig. 2, we impose the following initial conditions (ICs) and boundary conditions (BCs):

IC: $V(x, y, t=0)=f(x, y)$;

$\mathrm{BC}: \quad V(0, y, t)=0, \quad V(a, y, t)=0, \quad V(x, 0, t)=0, \quad$ and $V(x, b, t)=0$.

Using separation of variables and orthogonality of sinusoids, we obtain

$$
V(x, y, t)=\sum_{n=1}^{\infty} \sum_{m=1}^{\infty} A_{m n} \sin \left(\frac{n \pi}{a} x\right) \sin \left(\frac{m \pi}{b} y\right) \exp \left(-\lambda^{2} D t\right)
$$

with $A_{m n}=A_{n} B_{m}$ and $f(x, y)=f_{1}(x) f_{2}(y)$, where

$$
\begin{aligned}
& \lambda=\left(\frac{n \pi}{a}\right)^{2}+\left(\frac{m \pi}{b}\right)^{2} ; \\
& A_{n}=\frac{2}{a} \int_{0}^{a} f_{1}(x) \sin \left(\frac{n \pi}{a} x\right) d x,
\end{aligned}
$$

and

$$
B_{m}=\frac{2}{b} \int_{0}^{b} f_{2}(y) \sin \left(\frac{m \pi}{b} y\right) d y .
$$

Similarly, when solving the diffusion equation with respect to $\nabla V=0$ boundary conditions on rectangular device geometry, the initial and boundary conditions that we use are

IC: $V(x, y, t=0)=f(x, y)$;

$\mathrm{BC}: \quad \partial /\left.\partial x V(x, y, t)\right|_{x=0}=0, \partial /\left.\partial x V(x, y, t)\right|_{x=a}=0$, $\partial /\left.\partial y V(x, y, t)\right|_{y=0}=0$, and $\partial /\left.\partial y V(x, y, t)\right|_{y=b}=0$.

Similar to the previous case, using separation of variables and orthogonality of cosines, we get 


$$
\begin{aligned}
V(x, y, t)= & \sum_{n=0}^{\infty} \sum_{m=0}^{\infty} A_{m n} \cos \left(\frac{n \pi}{a} x\right) \cos \left(\frac{m \pi}{b} y\right) \\
& \times \exp \left(-\lambda^{2} D t\right),
\end{aligned}
$$

with $A_{m n}=A_{n} B_{m}, f(x, y)=f_{1}(x) f_{2}(y)$ and $\lambda$ is as given in Eq. (5), where

$$
\begin{aligned}
& A_{0}=\frac{1}{a} \int_{0}^{a} f_{1}(x) d x \quad(\text { for } n=0), \\
& A_{n}=\frac{2}{a} \int_{0}^{a} f_{1}(x) \cos \left(\frac{n \pi}{a} x\right) d x \quad(\text { for } n>0), \\
& B_{0}=\frac{1}{b} \int_{0}^{b} f_{2}(y) d y \quad(\text { for } m=0),
\end{aligned}
$$

and

$$
B_{m}=\frac{2}{b} \int_{0}^{b} f_{2}(y) \cos \left(\frac{m \pi}{b} y\right) d y \quad(\text { for } m>0) .
$$

Last, we present the solutions of the diffusion equation on circular device geometry sketched in Fig. 2. When solving the diffusion equation for the circular $V=0$ boundaries, we use the following boundary and initial conditions (conveniently presented in a cylindrical coordinate system):

IC: $V(r, \phi, t=0)=f(r, \phi)$ and BC: $V(r=a, \phi, t)=0$.

Using separation of variables and orthogonality of sines, cosines, and Bessel functions, we arrive

$$
V(r, t)=\sum_{m=1}^{\infty} A_{m} J_{0}\left(\frac{p_{0 m}}{a} r\right) \exp \left(-\frac{p_{0 m}^{2}}{a^{2}} D t\right)
$$

and

$$
A_{m}=\frac{2}{\left[a J_{1}\left(p_{0 m}\right)\right]^{2}} \int_{0}^{a} f(r) J_{0}\left(\frac{p_{0 m}}{a} r\right) r d r .
$$

Here we consider the zeroth-order Bessel solution for the circularly symmetric case where $V$ is just a function of $r$ and $t$, and $f$ is a function of $r$ because we use circularly symmetric Gaussian optical input beam to operate the diffusive conduction based switches.

To understand the characteristic voltage diffusion behavior of a diffusive conduction based switch for the three different types of boundary conditions (no boundary, $V=0$, and $\nabla V=0$ ), we investigate the voltage decay through diffusive conduction, for example, for an optically induced voltage buildup of $1 \mathrm{~V}$ on a rectangular $30 \times 30 \mu \mathrm{m}^{2}$ switch. With such a choice of using a smaller mesa switch, it is possible to enhance and display the effect of the boundary conditions, as shown in Fig. 3.

Figure 3 shows three plots of the diffusive voltage decay (a) first at the center of the switch, (b) then at a point very near to the switch metal contact, and (c) finally at the midpoint between the switch center and contact, with each plot superposing the time traces of the voltage decay for the three boundary conditions. Here while studying the diffusive conduction solutions for $V=0$ and $\nabla V=0$ boundary conditions,
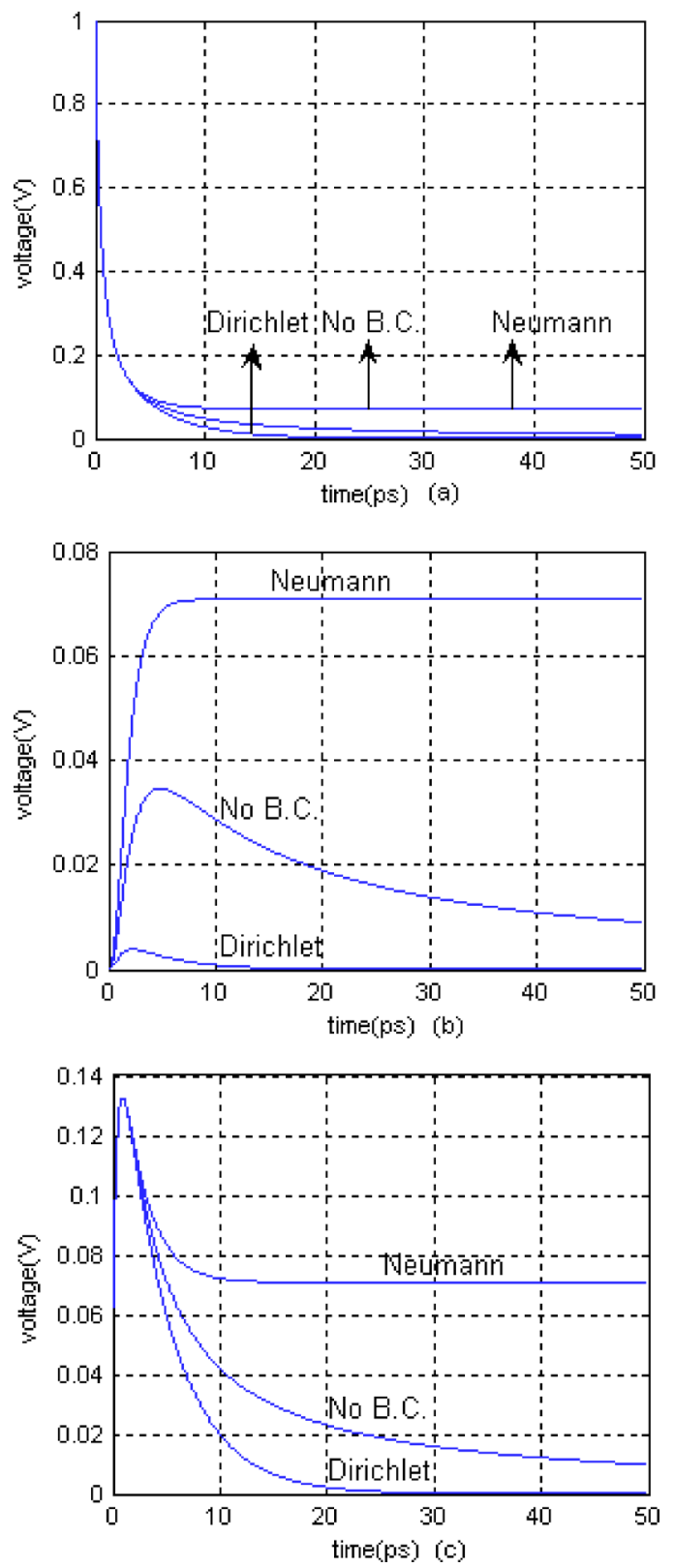

FIG. 3. Diffusive conductive voltage decay due to the three different boundary conditions (no boundary, $V=0$, and $\nabla V=0$ ): (a) at the center of the switch, (b) at a point very near to the switch contact line, and (c) at the midpoint between the switch center and contact.

we consider only the first 100 terms of the infinite series of Eqs. (4) and (8). The inclusion of 100 terms in the series suffices to converge a solution with a $0.5 \%$ error at $t=0$, and this error further decreases away rapidly over time (since the decay constant $\lambda^{2}$ is larger for the latter terms).

The $V=0$ boundary conditions provide the fastest voltage decay. This is because this type of boundary conditions pins the voltage at $0 \mathrm{~V}$ along the device boundaries. Furthermore, we observe that the device boundary conditions significantly affect the voltage decay at points later in time and/or closer to the device boundaries. This justifies our choice of using a smaller mesa to investigate the effect of boundary conditions.

To study the real-world voltage time traces of such a diffusive conduction based switch, we present the overall 


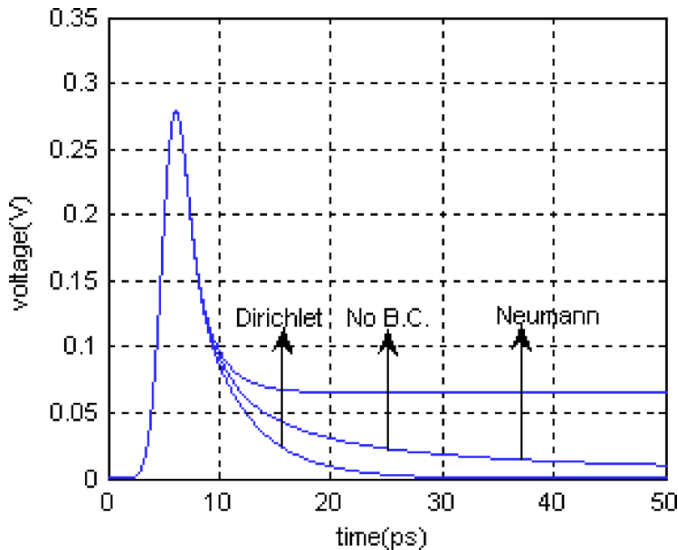

FIG. 4. Total voltage change for the three different boundary conditions (no boundary, $V=0$, and $\nabla V=0)$ at the center of the switch.

switch voltage profile that also includes the optically induced voltage buildup. Convolving the voltage decay with the incident optical pulse and the resulting voltage buildup (due to the vertical separation of the photogenerated carriers) in the case of small-signal analysis, we obtain the time traces plotted for the switch center in Fig. 4. The effects of boundary conditions on the voltage decay are fully transferred to the total voltage behavior. The $V=0$ boundary conditions expectedly yield the fastest switch recovery. This shows the significance of the device operation conditions. Although the external circuit does not predominantly determine the switching bandwidth, it is vital to properly bias the device. The external circuit is critical in removing the photogenerated charges. Thus, it matters to dc bias the device, which corresponds physically to short circuiting the device contact lines in the transient case and mathematically to the $V=0$ boundary conditions. In the case of not biasing the device, the device contact lines are left open circuit and the diffusive conduction process consequently slows down significantly.

\section{DIFFUSIVE CONDUCTIVE SWITCHING}

To understand the device operation in optical links, we first examine the steady state behavior when a train of control pulses is incident on the switch. When there is no control pulse (i.e., when a binary " 0 " is incident), the device response is simply flat and no change occurs to the probe pulse. In the other case, when there is a control pulse (i.e., when a binary "1" is incident), the device exhibits a switching response. When there is a train of control pulses, the switch goes through successive turn on and off periods. Using the analytical voltage decay expression we derive for a Gaussian initial condition, we calculate the device response to a sequence of control pulses as the sum of their voltage expressions all shifted by the pulse period with respect to the previous pulse. This sum is a series sum, and the terms of this sum all contain a $1 / t$ time dependence. This type of series sum always diverges to infinity. This can also be found in the simulations of previous work on this subject. ${ }^{25}$ Also, a series sum of $1 / t^{k}$, where $k>1$, always converges to a finite value. Since a $V=0$ boundary condition solution results in a voltage decay function that decreases faster than the case of no boundary, we can safely assume that the $V=0$ boundary
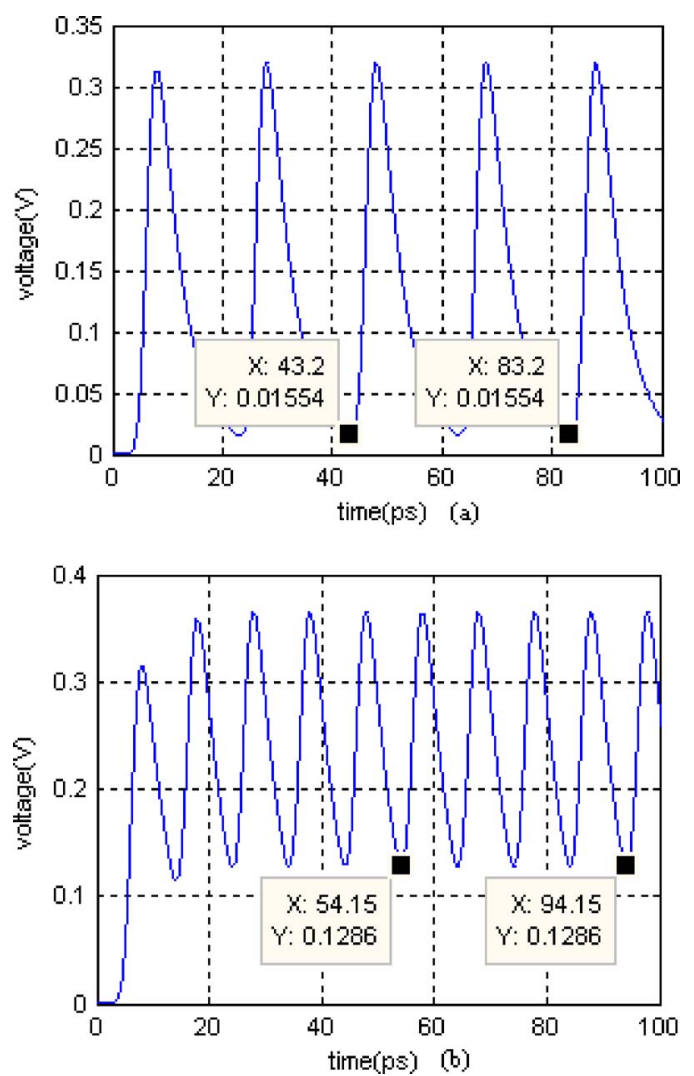

FIG. 5. Steady state behavior of a pulse train having a period of (a) 20 and (b) $10 \mathrm{ps}$. It is clear that both pulse trains reach steady state. When the period is smaller, the device reaches the steady state later and at a higher steady state value.

condition solution can be approximately modeled as the no boundary condition solution with $k>1$. This qualitative model reasonably suggests that the solution with $V=0$ boundary conditions always provides a finite steady state value that depends on the pulse period. Simulations representing this fact are presented for two different periods in Fig. 5. (Here note that since $\nabla V=0$ boundary conditions result in the accumulation of photogenerated charges, we know that it is not possible to reach a steady state value in the case of not biasing the device.)

In order to use such a diffusive conduction based optoelectronic switch for optical links, the device performance must be examined for basic data coding schemes. We present the eye diagrams of this device for the cases of both no boundary and $V=0$ boundary condition for return-to-zero in Fig. 6 and non-return-to-zero schemes in Fig. 7, all for optical links at 40 Gbps.

As clearly demonstrated in Figs. 6 and 7, V=0 boundary conditions provide a significant improvement for the eye diagrams. This type of boundary conditions leads to a stronger decaying behavior, which quickly abates the effect of previous bits.

One of the important ideas on the use of diffusive conduction based switches is their multichannel operation. Our typical fabricated devices have the dimensions of 300 $\times 300 \mu \mathrm{m}^{2}$. Since the light beams we are using typically have a full width at half maximum (FWHM) of about $7.5 \mu \mathrm{m}$, most of the device area is not directly used. Here we 

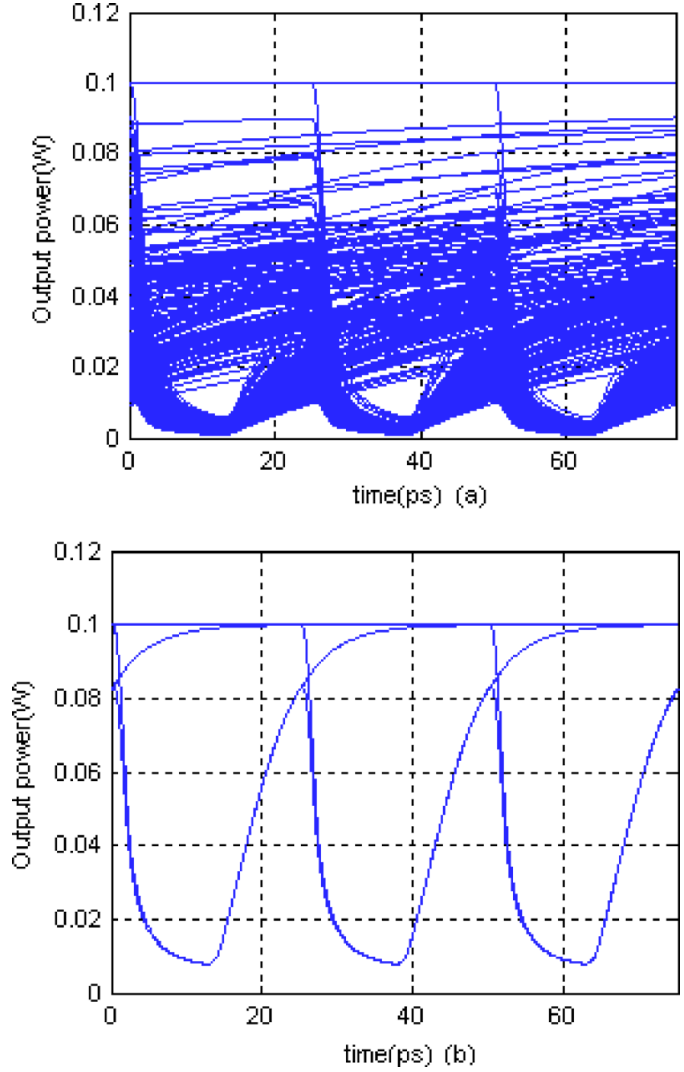

FIG. 6. The eye diagrams for a return-to-zero (RZ) optical link at $40 \mathrm{Gbps}$ : (a) with no boundary conditions and (b) with $V=0$ boundary condition. The improvement resulting from the $V=0$ boundary condition is clearly evident on the opening of the eye diagrams.

introduce a single ultrafast diffusive conduction based optoelectronic switch for multichannel applications that accommodates $>100$ optical channels with a channel density of 2000 channels per $1 \mathrm{~mm}^{2}$ of lateral device area and $<10 \%$ crosstalk between channels. Our $300 \times 300 \mu \mathrm{m}^{2}$ device with a switching bandwidth of $>50 \mathrm{GHz}$ therefore has, in principal, an aggregate transmission rate of $5 \mathrm{~Tb} / \mathrm{s}$. In such a device, all optical channels are operated independently of each other. Each input optical channel optically induces a local voltage change due to the voltage screening of its photogenerated carriers in its vicinity, which changes the absorption level through the switch for the output optical channel; this voltage change, in turn, quickly diffuses away across the lateral plane of the switch via diffusive conduction based on the internal local device $R C$ time constant. For multichannel operation, our approach uses a single optoelectronic switch, which is completely opposite to the traditional idea of arraying many switches. Consequently, this scheme eliminates the need for switch integration as well as the need for the precise alignment of the integrated individual switches or the optical channels. In this technique, the accommodation of multiple channels on a single switch is possible due to the unique, local behavior of diffusive conduction. In such diffusive conduction based optoelectronic switches, only the small portion of the device dictated by the spot size of the optical channel is switched and, thus, many optical channels may be run in parallel normal to a switch as long as they are sufficiently far away from each other. Although our approach avoids the
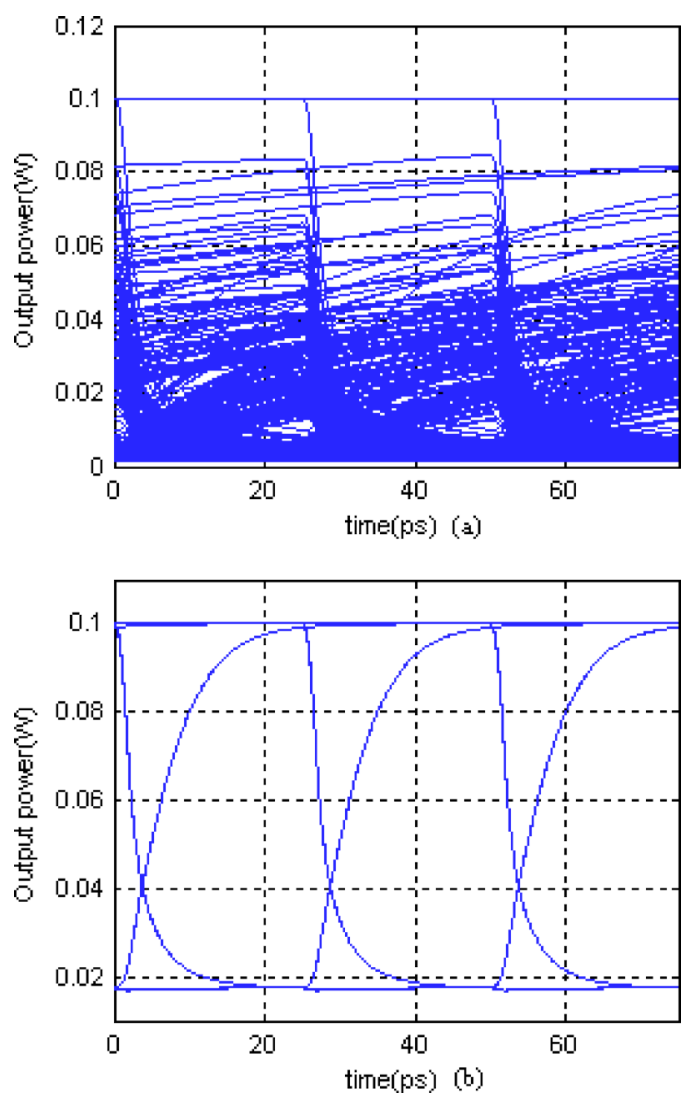

FIG. 7. The eye diagrams for a non-return-to-zero optical link at $40 \mathrm{Gbps}$ : (a) with no boundary conditions and (b) with $V=0$ boundary condition. Similarly, the $V=0$ boundary condition significantly improves the eye diagrams.

need to create parallel arrays of switches, it introduces technical challenges such as device heating and delivering multiple optical links to the switch.

Figure 8 shows the multichannel operation of the same diffusive conduction based optoelectronic switch as a function of channel spacing (and channel density) for different device $D$ constants (in $\mu \mathrm{m}^{2} / \mathrm{ps}$ ). We observe that the crosstalk between the parallel optical channels increases with

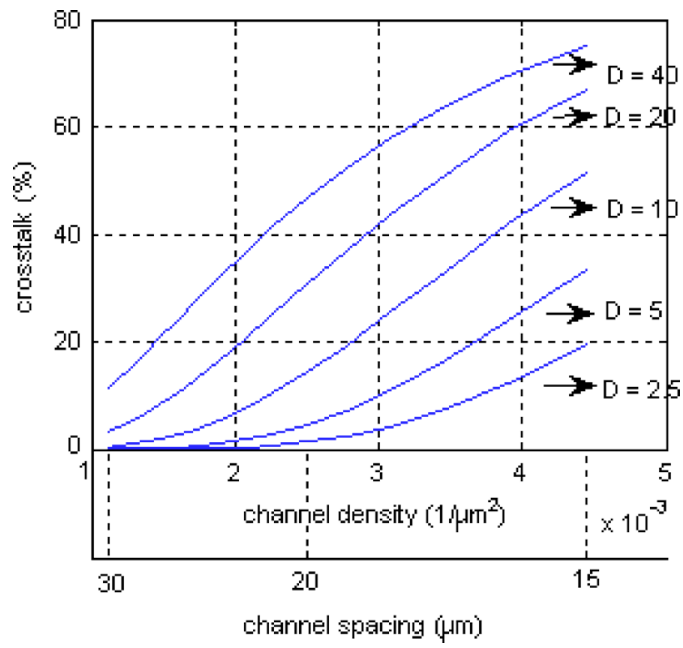

FIG. 8. Multichannel operation. The crosstalk is plotted as a function of channel spacing (and channel density) for different device diffusive constants $D$ (in $\left.\mu \mathrm{m}^{2} / \mathrm{ps}\right)$. 
the decreasing channel spacing (and the increasing channel density) as well as with the increasing switch speed. Considering the worst case for the crosstalk calculations even for the switches with high extinction ratio $(10 \mathrm{~dB})$ and no boundary conditions imposed on the switching, we observe that it is possible to establish multiple optical channels with a density of $2 \mu \mathrm{m}^{-2}$ for $D=10 \mu \mathrm{m}^{2} / \mathrm{ps}$ yielding $<10 \%$ cross-talk between the channels. This makes $>100$ optical channels on a $300 \times 300 \mu \mathrm{m}$ switch device, with a switching bandwidth of $50 \mathrm{GHz}$, apparently quite feasible. For the low cross-talk region $(<10 \%)$, the slope of the crosstalk curves over the channel spacing is shallow, because there the crosstalk contribution from the adjacent neighbors is infinitesimal.

In single channel operation, the voltage laterally diffuses across the length of the device. The overall effect on a given channel surrounded by multiple evenly spaced channels is roughly equivalent to reducing the effective length of the device to that of the spacing between the adjacent channels. A $20 \mu \mathrm{m}$ channel spacing becomes equivalent to a device area of $20 \times 20 \mu \mathrm{m}^{2}$. A channel with a $3 \times 3 \mu \mathrm{m}^{2}$ spot size can thus have its voltage diffuse by roughly a factor of 50 even within this much smaller area. This helps explain the low crosstalk in Fig. 8.

\section{CONCLUSIONS}

In this work, we studied the diffusive conduction that rapidly decays optically induced local voltage changes on optoelectronic switches in a picosecond time scale, allowing for exceptionally high switching bandwidths. Here we present the characteristic Green's function of the diffusive conduction derived for arbitrary initial conditions. We also report on the series solutions of the diffusive conduction obtained for different boundary conditions $(V=0$ and $\nabla V=0$ along the device boundaries at all times) and different mesa geometries (rectangular and circular). Using these analytical results, we investigate the effect of boundary conditions on the switching operation and the steady state behavior of such diffusive conduction based optoelectronic switches. We demonstrate the feasibility of using these optoelectronic switches to establish $40 \mathrm{~Gb} / \mathrm{s}$ optical links. Furthermore, we introduce the multichannel operation of a single diffusive conduction based switch for high aggregate transmission. We predict the possibility of transmitting at a rate of $>5 \mathrm{~Tb} / \mathrm{s}$ by accommodating $>100$ channels on a single device of $50 \mathrm{GHz}$ switching bandwidth.
${ }^{1}$ B. Xiong, J. Wang, L. Zhang, J. Tian, C. Sun, and Y. Luo, IEEE Photonics Technol. Lett. 17, 327 (2005).

${ }^{2}$ A. Vonsovici and L. Vescan, IEEE J. Sel. Top. Quantum Electron. 4, 1011 (1998).

${ }^{3}$ V. A. Sabnis et al., Appl. Phys. Lett. 83, 469 (2004).

${ }^{4}$ I. Kimukin, N. Biyikli, T. Kartaloglu, O. Aytur, and E. Ozbay, IEEE J. Sel. Top. Quantum Electron. 10, 766 (2004).

${ }^{5}$ T. Kawanishi, K. Higuma, T. Fujita, J. Ichikawa, T. Sakamoto, S. Shinada, and M. Izutsu, J. Lightwave Technol. 23, 87 (2005).

${ }^{6}$ H. V. Demir, V. A. Sabnis, O. Fidaner, J. S. Harris, Jr., D. A. B. Miller, and J.-F. Zheng, Opt. Express 12, 310 (2004).

${ }^{7}$ S. Z. Zhang, Y.-J. Chiu, P. Abraham, and J. E. Bowers, IEEE Photonics Technol. Lett. 11, 191 (1999).

${ }^{8}$ G. L. Li, C. K. Sun, S. A. Pappert, W. X. Chen, and P. K. L. Yu, IEEE Trans. Microwave Theory Tech. 47, 1177 (1999).

${ }^{9}$ G. L. Li, S. A. Pappert, P. Mages, C. K. Sun, W. S. C. Chang, and P. K. L. Yu, IEEE Photonics Technol. Lett. 13, 1076 (2001).

${ }^{10}$ K. Kubota, J. Noda, and O. Mikami, IEEE J. Quantum Electron. 16, 754 (1980).

${ }^{11}$ T. Ido, S. Tanaka, M. Suzuki, M. Koizumi, H. Sano, and H. Inoue, J. Lightwave Technol. 14, 2026 (1996).

${ }^{12}$ Y.-J. Chiu, H.-F. Chou, V. Kaman, P. Abraham, and J. E. Bowers, IEEE Trans. Microwave Theory Tech. 14, 792 (2002).

${ }^{13}$ Y. Akage, K. Kawano, S. Oku, R. Iga, H. Okamoto, Y. Miyamoto, and H. Takeuchi, Electron. Lett. 37, 299 (2001).

${ }^{14}$ S. Matsuura, G. A. Blake, R. A. Wyss, J. C. Pearson, C. Kadow, A. W. Jackson, and A. C. Gossard, Appl. Phys. Lett. 74, 2872 (1999).

${ }^{15}$ V. M. Hietala, G. A. Vawter, T. M. Brennan, and B. E. Hammons, IEEE Trans. Microwave Theory Tech. 43, 2291 (1995).

${ }^{16}$ K. S. Giboney, M. J. W. Rodwell, and J. E. Bowers, IEEE Trans. Microwave Theory Tech. 45, 1310 (1997).

${ }^{17}$ Y.-J. Chiu, S. B. Fleischer, D. Lasaosa, and J. E. Bowers, Appl. Phys. Lett. 71, 2508 (1997).

${ }^{18}$ J. E. Bowers and C. A. Burrus, J. Lightwave Technol. 5, 1339 (1987).

${ }^{19}$ S. Kodama, T. Yoshimatsu, and H. Ito, Electron. Lett. 40, 1 (2004).

${ }^{20}$ K. S. Giboney, Ph.D. dissertation, University of California, Santa Barbara, 1995.

${ }^{21}$ S. Zhang, Ph.D. dissertation, University of California, Santa Barbara, 1999.

${ }^{22}$ M. B. Yairi, C. W. Coldren, D. A. B. Miller, and J. S. Harris, Jr., Appl. Phys. Lett. 75, 597 (1999).

${ }^{23}$ M. B. Yairi, H. V. Demir, P. B. Atanackovic, and D. A. B. Miller, IEEE J. Quantum Electron. 40, 143 (2004).

${ }^{24}$ V. A. Sabnis, H. V. Demir, M. B. Yairi, J. S. Harris, Jr., and D. A. B. Miller, J. Appl. Phys. 95, 2258 (2004).

${ }^{25}$ M. B. Yairi, H. V. Demir, and D. A. B. Miller, Opt. Quantum Electron. 33, 1035 (2001).

${ }^{26}$ M. B. Yairi and D. A. B. Miller, J. Appl. Phys. 91, 4374 (2002)

${ }^{27}$ D. Streb, M. Vitzethum, P. Kiesel, G. H. Dohler, and M. Kneissl, Superlattices Microstruct. 25, 21 (1999).

${ }^{28}$ H. Schneider, E. C. Larkins, J. D. Ralston, J. Fleissner, G. Bender, and P. Koidl, Appl. Phys. Lett. 60, 2648 (1992).

${ }^{29}$ P. J. Bradley, C. Rigo, and A. Stano, IEEE J. Quantum Electron. 32, 43 (1996).

${ }^{30}$ M. Ershov, Appl. Phys. Lett. 72, 2865 (1998).

${ }^{31}$ G. Livescu, D. A. B. Miller, T. Sizer, D. J. Burrows, J. E. Cunningham, A. C. Gossard, and J. H. English, Appl. Phys. Lett. 54, 748 (1989).

${ }^{32}$ M. B. Yairi, Ph.D. dissertation, Stanford University, 2001.

${ }^{33}$ D. A. B. Miller, D. S. Chemla, T. C. Damen, A. C. Gossard, W. Wiegmann, T. H. Wood, and C. A. Burrus, Phys. Rev. Lett. 53, 2173 (1984). 